\title{
Estimation of Analog Parametric Test Metrics Using Copulas
}

\author{
Ahcène Bounceur, Member, IEEE, Salvador Mir, Member, IEEE and \\ Haralampos-G. Stratigopoulos, Member, IEEE
}

\begin{abstract}
A new technique for the estimation of analog parametric test metrics at the design stage is presented in this paper. This technique employs the copulas theory to estimate the distribution between random variables that represent the performances and the test measurements of the circuit under test (CUT). A copulas-based model separates the dependencies between these random variables from their marginal distributions, providing a complete and scale-free description of dependence that is more suitable to be modeled using well-known multivariate parametric laws. The model can be readily used for the generation of an arbitrarily large sample of CUT instances. This sample is thereafter used for estimating parametric test metrics such as defect level (or test escapes) and yield loss. We demonstrate the usefulness of the proposed technique to evaluate a Built-In-Test (BIT) technique for an RF Low Noise Amplifier (LNA) and to set test limits that result in a desired trade-off between test metrics. In addition, we compare the proposed technique with previous ones that rely on direct density estimation.
\end{abstract}

\section{INTRODUCTION}

A major hurdle when dealing with newly proposed analog tests is the difficulty to evaluate them at the design stage. This evaluation is typically carried out in terms of incurred costs, such as those due to the silicon overhead and the degradation of performances of the CUT in the case of design-for-test (DfT) techniques, and those due to the probabilities of test errors. Test errors are typically described by test metrics such as defect level (probability that a device that passes the test is faulty) and yield loss (probability that a functional device fails the test). While silicon overhead and performance degradation can be easily estimated by designers, the estimation of analog test metrics is difficult to obtain.

In digital circuits, signals are robust with respect to parametric deviations since only discrete-time binary values are considered. As a result, test metrics for digital circuits are essentially evaluated with respect to the presence of fabrication defects that lead typically to catastrophic faults, whereas parametric deviations are overlooked. On the other hand, analog devices, including mixed-signal, RF or microelectromechanical devices, as well as digital devices that operate at very high frequencies, are very much sensitive to

A. Bounceur is with Université Européenne de Bretagne, Brest, France (email: Ahcene.Bounceur@univ-brest.fr).

S. Mir and H.-G. Stratigopoulos are with TIMA Laboratory (CNRSGrenoble Institute of Technology-Université Joseph Fourier), Grenoble, France (e-mail: \{Salvador.Mir,Haralampos.Stratigopoulos\}@imag.fr).

This work has been carried out in the frame of the European MEDEA+ project NanoTest.

A preliminary version of this work appears in the proceedings of the IEEE International Mixed-Signals, Sensors and Systems Test Workshop, 2008 [1]. parametric deviations. In this case, signals are continuous in time and value and, thereby, they are dependent on parametric deviations originated by the manufacturing process. As opposed to the case of digital circuits, the estimation of parametric test metrics is essential for these devices. For example, an analog BIT technique with its associated test limits must be designed so that it adds a minimum parametric yield loss while it detects a maximum of devices out of specifications. In other words, BIT measurements and their associated test limits must be selected in such a way that test metrics are optimized.

Test escape is typically a low probability event given that a mature fabrication process will result in a low number of defective parts per million (dppm), typically in the order of a few hundreds. Similarly, yield loss is a low probability event given a well-designed test methodology. Therefore, to achieve a parts per million (ppm) precision for the estimation of parametric test metrics, it is necessary to generate in a natural way a population of at least one million circuits and to obtain their circuit performances and test measurements (hereafter jointly called output parameters). Projecting test escape and yield loss at ppm levels using a smaller set of circuits will certainly lead to erroneous judgements about the quality of tests.

The output parameters depend on circuit parameters, namely design and technology parameters, as well as on input stimuli and environmental conditions. The circuit parameters do not have deterministic values, but are random variables that follow statistical distributions centered at the nominal values. These statistical distributions are known a priori and are included in the process design kit. Therefore, we can easily rely on a Monte Carlo analysis to create one million circuit netlists that conform to the underlying distribution of circuit parameters. Accordingly, output parameters are random variables as well, yet their joint distribution is initially unknown. To compute the output parameters of the one million circuits and, thereby, to compute the test metrics, we will need to simulate the netlists. However, in practice, we can only afford to run a few simulations. Clearly, simulating one million netlists is unfeasible which points to the need of using instead of circuit simulation a statistical model of the CUT that describes accurately the relationships between the output parameters.

An idealized formulation of the problem is as follows. Let $\mathbf{X}=\left(X_{1}, X_{2}, \ldots, X_{n}\right)$ denote the vector that represents $n$ output parameters $X_{i}, i=1, \ldots, n$. Suppose that we run $m$ circuit simulations to obtain $m$ observations of $\mathbf{X}$. Starting from these $m$ observations, how can we build a statistical 
model of the CUT to generate an additional sample of $N$ observations, where $N \gg m$, without relying on circuit simulation? In essence, this sample would correspond to the output parameters of $N$ new circuit instances. Once it is generated, it can be readily used to compute parametric test metrics based on event counting and relative frequencies. For example, out of the $N$ circuit instances, suppose that $N_{p t}$ pass the test and $N_{f p t}$ are faulty and pass the test. Then, we can express test escape as $T_{E}=N_{f p t} / N_{p t}$. Similarly, if $N_{f}$ circuit instances out of $N$ are functional and $N_{f f t}$ are functional and fail the test, then yield loss can be expressed as $Y_{L}=N_{f f t} / N_{f}$.

Previous methods to estimate parametric test metrics rely on estimating the joint multivariate probability density function (PDF), $f(x)$, of the output parameters [2], [3]. The density $f(x)$ represents amply the dependency between the output parameters and, in essence, forms a statistical model of the CUT that can effectively replace circuit-level simulations. In particular, it can be simulated (or sampled) to generate direct observations of $\mathbf{X}$ corresponding to new CUT instances, as opposed to creating circuit netlists through a Monte Carlo approach and simulating each netlist at transistor-level using different test benches corresponding to each $X_{i}, i=1, \ldots, n$. Density simulation can be carried out extremely fast compared to circuit-level simulations. As will be explained in greater detail in Section II, the problem with this approach is that the accurate estimation of a multivariate density becomes increasingly troublesome as the number of output parameters increases.

In this paper, we propose to construct a statistical model of the CUT using the copulas theory. This approach boils down to estimating a density as before, but this time, density estimation is attempted in a different space where the marginal distributions of output parameters are dissociated from their dependencies. In this way, we circumvent the curse of dimensionality problems that we encounter when a density is fitted in the original multivariate space $X$. As before, the copulas theory can be used to generate an arbitrary sample of circuit instances through which the estimation of test metrics becomes simple and effortless.

The structure of the paper is as follows. We will first review in more detail some previous works in this field in Section II. Section III will introduce some basic concepts that are used in the copulas theory. Next, Section IV will introduce formally the copulas theory. The procedure for analog test metrics estimation will be summarized in Section V. The use of copulas-based statistical models for the estimation of parametric test metrics will be illustrated for a BIT technique of an $1.9 \mathrm{GHz}$ cascode LNA in Section VI. In Section VII, we will give recommendations as to which statistical model should be used in different scenarios. Finally, Section VIII will conclude the paper.

\section{Previous Work}

Regression modeling is one of the first methods proposed to alleviate the burden of Monte Carlo circuit simulations [4]. Let $\mathbf{P}$ denote the vector of circuit parameters. We first sample the circuit parameter space using design of experiments, such that the selected sample, denoted by $\mathbf{P}^{1}, \mathbf{P}^{2}, \ldots, \mathbf{P}^{m}$, spans uniformly the entire circuit parameter space. We simulate each sample of circuit parameters to obtain the corresponding output parameters, denoted by $\mathbf{X}^{1}, \mathbf{X}^{2}, \ldots, \mathbf{X}^{m}$. Then, the data $\left\{\left(\mathbf{P}^{i}, \mathbf{X}^{i}\right)\right\}_{i=1}^{m}$ is used to train regression functions $f_{j}$ that map any point in the circuit parameter space to the output parameter space, i.e. $f_{j}: \mathbf{P} \rightarrow X_{j}$, where $X_{j}$ denotes the $j$-th output parameter. Once the regression functions are trained, we can use probability sampling to sample $\mathbf{P}^{i}, i=1, \ldots, N$, $N \gg 1$, and then obtain $\mathbf{X}^{i}$ through the regression functions. This method can also be applied in multiple steps through the architectural hierarchy of the circuit using intermediate behavioral models [5]. The major difficulty of this approach lies on the regression and behavioral modeling. In particular, it is difficult to build regression functions when a large number of circuit parameters have a degree of influence that cannot be ignored. On the other hand, behavioral modeling might not capture all non-ideal effects that give rise to faulty circuits. This method has been largely explored in the field of yield modeling and yield optimization.

Another possibility, as mentioned in the introduction, is to estimate an analytical expression of the joint PDF of output parameters $\mathbf{X}=\left(X_{1}, X_{2}, \ldots, X_{n}\right)$, denoted here by $f\left(x_{1}, x_{2}, \ldots, x_{n}\right)$. When the number of output parameters is small, the joint PDF can be integrated to directly calculate the test metrics. For example, for the case of one performance $X_{1}$ with specifications $\left[s_{1}, s_{2}\right]$ and one test measurement $X_{2}$ with test limits $\left[t_{1}, t_{2}\right]$, the test escape can be expressed as

$$
T_{E}=\int_{-\infty}^{s_{1}} \int_{t_{1}}^{t_{2}} f\left(x_{1}, x_{2}\right) d x_{2} d x_{1}+\int_{s_{2}}^{+\infty} \int_{t_{1}}^{t_{2}} f\left(x_{1}, x_{2}\right) d x_{2} d x_{1} .
$$

Alternatively, if direct integration is not possible, we can rely on probability sampling to generate a large number of observations $\mathbf{X}^{i}, i=1, \ldots, N, N \gg 1$, from $f\left(x_{1}, x_{2}, \ldots, x_{n}\right)$. There are different algorithms for sampling a multivariate density, such as Slice Sampler that belongs to the family of Monte Carlo Markov Chain (MCMC) algorithms. The method uses the process design kit of the CUT and, therefore, it is very easy to automate. Its major difficulty lies on the estimation of the joint PDF itself.

Parametric PDF estimation using a multivariate Gaussian law has been considered in [2]. The statistical parameters of this law include the mean vector and the variance-covariance matrix of the output parameters. These statistical parameters can be easily calculated based on an initial sample of circuits generated by Monte Carlo simulation. This model is accurate only if each output parameter follows a Gaussian distribution and all output parameters are linearly correlated.

Non-parametric PDF estimation using an adaptive Kernel Density Estimator (KDE) has been considered in [3]. This method provides a more intuitive way to estimate the underlying PDF by revoking the assumption that it follows a specific parametric form (e.g. Gaussian). However, it depends on an auxiliary smoothing parameter, called bandwidth, which defines the weight that is given at the tails of the PDF where rare events (e.g. test escape and yield loss) occur. The higher 
the number of output parameters is, the more likely it is that the density at the tails will be overestimated or underestimated, resulting in inaccurate estimates of test metrics. In short, this method is useful to compare test measurements based on the resulting test metrics, yet the test metrics predictions cannot be trusted in absolute terms. Practically, accurate predictions can only be obtained when dealing with a few performances and test measurements.

\section{BASIC CONCEPTS}

In this Section, we will introduce some basic concepts that will be used in the rest of the paper. These concepts include the dependence and rank correlation of a pair of random variables, the inverse transform sampling to generate samples at random from a probability distribution given its cumulative distribution function (CDF), and the Kolmogorov-Smirnov (KS) goodness-of-fit test.

\section{A. Dependence and rank correlation}

Different measures are used in order to define the relationship between two continuous random variables $X$ and $Y$ [6]. Typically, this relationship is measured by the Pearson correlation factor $\rho_{X Y}=\operatorname{cov}(X, Y) /\left(\sigma_{X} \sigma_{Y}\right)$, where $\operatorname{cov}(X, Y)$ is the covariance of $X$ and $Y$ and $\sigma_{X}, \sigma_{Y}$ are the standard deviations of $X$ and $Y$, respectively. If the variables are independent, $\rho_{X Y}=0$. However, the converse is not true, since the Pearson correlation factor is only sensitive to the linear relationship between the two variables. In general, it cannot provide an idea of the intensity of the link that exists between them. Moreover, in the presence of outliers, it may give a completely misleading impression of correlation.

Nonparametric statistics such as rank correlation coefficients are more robust than the Pearson correlation factor. Basically, they measure the extent to which one variable tends to increase as the other one increases, without requiring the increase to be represented by a linear relationship. To describe rank correlation coefficients, the notion of concordance must be defined. Let $\left(X^{1}, Y^{1}\right), \ldots,\left(X^{j}, Y^{j}\right)$ be a random sample of $m$ observations of $(X, Y)$. Then,

- $\left(X^{i}, Y^{i}\right)$ and $\left(X^{j}, Y^{j}\right)$ are said to be concordant if $\left(X^{i}-\right.$ $\left.X^{j}\right)\left(Y^{i}-Y^{j}\right)>0$.

- $\left(X^{i}, Y^{i}\right)$ and $\left(X^{j}, Y^{j}\right)$ are said to be discordant if $\left(X^{i}-\right.$ $\left.X^{j}\right)\left(Y^{i}-Y^{j}\right)<0$.

Notice that the Pearson correlation factor $\rho_{X Y}$ is not a concordance measure. The two main used rank correlation coefficients are the Kendall's $\tau$ [7] and the Spearman's $\rho_{S}$ [8]. Herein, we will use the Kendall's $\tau$. The sample version of the Kendall's $\tau$ is defined as follows. There are $\left(\begin{array}{c}m \\ 2\end{array}\right)$ distinct pairs $\left(X^{i}, Y^{i}\right)$ and $\left(X^{m}, Y^{m}\right)$ of observations in the sample, and each pair is either concordant or discordant. The sample estimator $\hat{\tau}_{X Y}$ is calculated as the relative frequency of concordant pairs minus the relative frequency of discordant pairs, i.e.

$$
\hat{\tau}_{X Y}=\frac{2}{m(m-1)} \sum_{i=1}^{m} \sum_{\substack{j=1 \\ j>i}}^{m} \operatorname{sgn}\left[\left(X^{i}-X^{j}\right)\left(Y^{i}-Y^{j}\right)\right],
$$

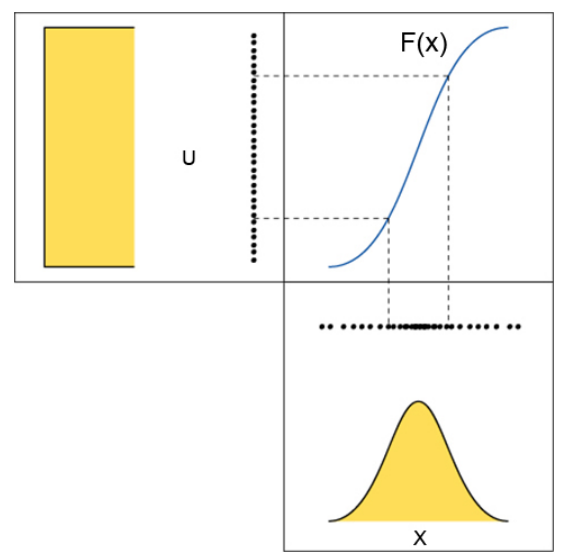

Fig. 1. The inverse method to generate a sample from a Gaussian distribution.

where

$$
\operatorname{sgn}(z)=\left\{\begin{array}{cc}
1 & \text { if } z \geq 0 \\
-1 & \text { if } z<0
\end{array}\right.
$$

Finally, it turns out that for the bivariate Gaussian distribution, the Kendall's $\tau$ can be calculated from the linear Pearson correlation coefficient as [9]

$$
\tau_{X Y}=\frac{2}{\pi} \arcsin \rho_{X Y} .
$$

For large $m$, it is preferable to calculate $\rho_{X Y}$ and then derive $\tau_{X Y}$ using Equation (2).

\section{B. Inverse transform sampling}

A classical approach for generating samples from an onedimensional CDF is the inverse transform sampling method. Given a continuous random variable $X$ with a CDF $F(x)=$ $P[X \leq x]$, the transform $U=F(X)$ results in a random variable $U$ that is uniform in $[0,1]$. Moreover, if $U$ has a uniform distribution in [0,1] and $X$ is defined as $X=F^{-1}(U)$, where $F^{-1}(u)=\inf \{x: F(x) \geq u\}$ and inf denotes the infimum (or greatest lower bound) of a set of real numbers, then the CDF of $X$ is $F(X)$. In order to generate an arbitrary large sample of $X$, we start with an uniformly distributed sample of $U$ that can easily be generated using a standard pseudo-random generator. For each sampled value of $U$, we can calculate a value of $\mathrm{X}$ using the inverse CDF given by $X=F^{-1}(U)$. Figure 1 illustrates this method for the case of a Gaussian random variable. If the function $F(x)$ is not invertible, other sampling methods can be considered [10], [11].

\section{Classical univariate goodness-of-fit test}

Many goodness-of-fit tests for copulas reduce the multivariate problem to an univariate one, and then apply an univariate test. Here, we will use the K-S test [12]. This test can be used to compare an empirical sample of size $m$ of an univariate distribution $\hat{F}(x)$ to a specific theoretical distribution $F(x)$ or to compare two empirical distribution samples of size $m$ to decide if they both come from the same distribution. 


\section{Copulas-Based Statistical Model}

\section{A. The basic idea}

Let $\mathbf{X}=\left(X_{1}, X_{2}, \ldots, X_{n}\right)$ denote a random vector of $n$ random variables $X_{1}, X_{2}, \ldots, X_{n}$ and let $f\left(x_{1}, x_{2}, \ldots, x_{n}\right)$ denote their joint PDF. Let also $f_{i}\left(x_{i}\right)$ and $F_{i}\left(x_{i}\right)$ denote respectively the marginal $\mathrm{PDF}$ and $\mathrm{CDF}$ of $X_{i}$. The usual approach to the problem is to first estimate the density $f\left(x_{1}, x_{2}, \ldots, x_{n}\right)$ and then simulate it to obtain the required number of random samples of $\mathbf{X}$ [2], [3]. If we transform $X_{i}$ according to their CDF, then we obtain $n$ random variables $U_{1}=F_{1}\left(X_{1}\right), U_{2}=F_{2}\left(X_{2}\right), \ldots, U_{n}=F_{n}\left(X_{n}\right)$ that follow a uniform distribution in $[0,1]$, as discussed in Section III-B. Since these transformations are invertible, specifying the dependency between the random variables $X_{i}$ is the same as specifying the dependency between the random variables $U_{i}$. Let now $c\left(u_{1}, u_{2}, \ldots, u_{n}\right)$ denote the joint PDF of $\mathbf{U}=$ $\left(U_{1}, U_{2}, \ldots, U_{n}\right)$. Instead of using probability sampling on $f\left(x_{1}, x_{2}, \ldots, x_{n}\right)$ to obtain samples of the random vector $\mathbf{X}$, we can achieve the same objective by first estimating $c\left(u_{1}, u_{2}, \ldots, u_{n}\right)$, subsequently using probability sampling on $c\left(u_{1}, u_{2}, \ldots, u_{n}\right)$ to obtain samples of the random vector $\mathbf{U}$, and finally using the inverse transformations $X_{i}=$ $F_{i}^{-1}\left(U_{i}\right)$. In this way, the problem of estimating the density $f\left(x_{1}, x_{2}, \ldots, x_{n}\right)$ has been converted to estimating a density $c\left(u_{1}, u_{2}, \ldots, u_{n}\right)$ that has uniform marginal distributions.

The benefit from the above transformation is that the density $c\left(u_{1}, u_{2}, \ldots, u_{n}\right)$ may have a known parametric form, even in cases where $f\left(x_{1}, x_{2}, \ldots, x_{n}\right)$ has a nonparametric form, i.e. a form that does not belong to any of the known families of distributions. In general, $f\left(x_{1}, x_{2}, \ldots, x_{n}\right)$ has a nonparametric form since the marginal distributions $f_{i}\left(x_{i}\right)$ have distinct parametric or even nonparametric forms. Therefore, given that nonparametric density estimation presents a lot of difficulties in high-dimensional spaces [13], this transformation may largely simplify the density estimation problem.

The CDF $C\left(u_{1}, u_{2}, \ldots, u_{n}\right)$ associated with the density $c\left(u_{1}, u_{2}, \ldots, u_{n}\right)$ is called copula ${ }^{1}$. In the following sections, we will introduce some properties of the copula function and we will focus on the copula that results from a standard multivariate Gaussian density $f\left(x_{1}, x_{2}, \ldots, x_{n}\right)$, that is the Gaussian copula. Then, we will give an example of a bivariate non-Gaussian density for which $f_{1}\left(x_{1}\right)$ is a Gamma distribution, $f_{2}\left(x_{2}\right)$ is a Gaussian distribution and their copula $C\left(u_{1}, u_{2}\right)$ is a Gaussian copula. Finally, we will show how to perform probability sampling on a Gaussian copula and how to test the goodness-of-fit of a Gaussian copula.

\section{B. Copulas theory}

Formally, a copula is a multivariate joint distribution defined on the $n$-dimensional unit cube $[0,1]^{n}$ such that every marginal

\footnotetext{
${ }^{1}$ The notion of copula was introduced by A. Sklar in 1959 [14] when studying the relationship between a multivariate PDF and its lower dimensional marginal distributions. Copulas were primarily used in the development of the theory of probabilistic metric spaces. Later, they were of interest to define nonparametric measures of dependence between random variables, and since then, they began to play an important role in probability and mathematical statistics. An introduction to copulas is given in [6]. Basic tutorials on copulas are given in software packages [10], [15].
}

distribution is uniform on the interval [0,1]. Specifically, $C$ : $[0,1]^{n} \rightarrow[0,1]$ is a $n$-copula if

- $C(\mathbf{u})=0, \forall \mathbf{u} \in[0,1]^{n}$ if $\exists k \in\{1, \ldots, n\}$ such that $u_{k}=0$ (i.e. if at least one component of the vector $\mathbf{u}$ is equal to 0$)$.

- $C(\mathbf{u})=u_{k}$ if all components of $\mathbf{u}$ are 1 except the $k$-th one which is equal to $u_{k}$.

- $C(\mathbf{u})$ is $n$-increasing, i.e. is increasing in each component $u_{k}$ with $k \in\{1,2, \ldots, n\}$.

One of the most relevant results in copulas theory is Sklar's theorem [6], [14]. Let $X_{1}, X_{2}, \ldots, X_{n}$ be $n$ random variables with $\mathrm{CDF} F_{1}\left(x_{1}\right), F_{2}\left(x_{2}\right), \ldots, F_{n}\left(x_{n}\right)$, respectively, and let $F\left(x_{1}, x_{2}, \ldots, x_{n}\right)$ denote their joint CDF. Sklar's theorem states that there exists a copula $C$ such that $\forall \mathbf{x}=$ $\left(x_{1}, x_{2}, \ldots, x_{n}\right) \in \mathbb{R}^{n}$

$$
F\left(x_{1}, \ldots, x_{n}\right)=C\left(F_{1}\left(x_{1}\right), \ldots, F_{n}\left(x_{n}\right)\right) .
$$

Equivalently, using $u_{i}=F_{i}\left(x_{i}\right), \forall \mathbf{u}=\left(u_{1}, u_{2}, \ldots, u_{n}\right) \in$ $[0,1]^{n}$ we can write

$$
C\left(u_{1}, \ldots, u_{n}\right)=F\left(F_{1}^{-1}\left(u_{1}\right), \ldots, F_{n}^{-1}\left(u_{n}\right)\right) .
$$

Moreover, if $F_{1}\left(x_{1}\right), F_{2}\left(x_{2}\right), \ldots, F_{n}\left(x_{n}\right)$ are continuous, then $C$ is unique.

Sklar's theorem expresses the basic idea of dependence modeling via copula functions, by stating that for any multivariate distribution, the univariate marginal distributions and their dependence structure can be separated, with the latter completely described by a copula function. By applying Sklar's theorem, we can easily derive the expression of the multivariate density $c\left(F_{1}\left(x_{1}\right), \ldots, F_{n}\left(x_{n}\right)\right)$ associated with a copula $C\left(F_{1}\left(x_{1}\right), \ldots, F_{n}\left(x_{n}\right)\right)$. Letting $f\left(x_{1}, x_{2}, \ldots, x_{n}\right)$ denote the joint PDF of the random vector $\mathbf{X}=\left(X_{1}, X_{2}, \ldots, X_{n}\right)$, we have

$$
\begin{aligned}
f\left(x_{1}, \ldots, x_{n}\right) & =\frac{\partial^{n} F\left(x_{1}, \ldots, x_{n}\right)}{\partial x_{1} \cdots \partial x_{n}} \\
& =\frac{\partial^{n}\left[C\left(F_{1}\left(x_{1}\right), \ldots, F_{n}\left(x_{n}\right)\right)\right]}{\partial F_{1}\left(x_{1}\right) \cdots \partial F_{n}\left(x_{n}\right)} \cdot \prod_{i=1}^{n} f_{i}\left(x_{i}\right) \\
& =c\left(F_{1}\left(x_{1}\right), \ldots, F_{n}\left(x_{n}\right)\right) \cdot \prod_{i=1}^{n} f_{i}\left(x_{i}\right)
\end{aligned}
$$

or

$$
c\left(F_{1}\left(x_{1}\right), \ldots, F_{n}\left(x_{n}\right)\right)=\frac{f\left(x_{1}, \ldots, x_{n}\right)}{\prod_{i=1}^{n} f_{i}\left(x_{i}\right)}
$$

\section{Gaussian copulas}

Let $f_{i}\left(x_{i}\right)$ be standard Gaussian distributions, i.e. $X_{i} \sim$ $N(0,1)$, and let $R$ be the correlation matrix with entries $R_{i j}=\hat{\tau}_{X_{i} X_{j}}, i, j=1, \ldots, n$. The resulting copula $C\left(u_{1}, u_{2}, \ldots, u_{n}\right)$ is called Gaussian copula. The density associated with $C\left(u_{1}, u_{2}, \ldots, u_{n}\right)$ is obtained using Equation (6) 


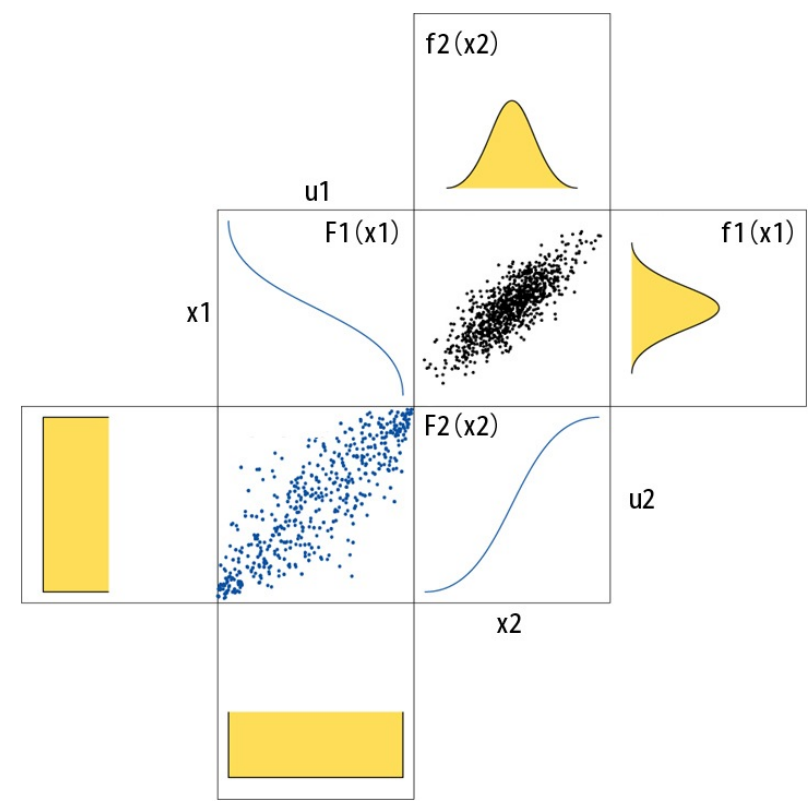

Fig. 2. Generation of a sample of a Gaussian copula from a sample of a bivariate standard Gaussian distribution with correlation coefficient $\rho=0.8$.

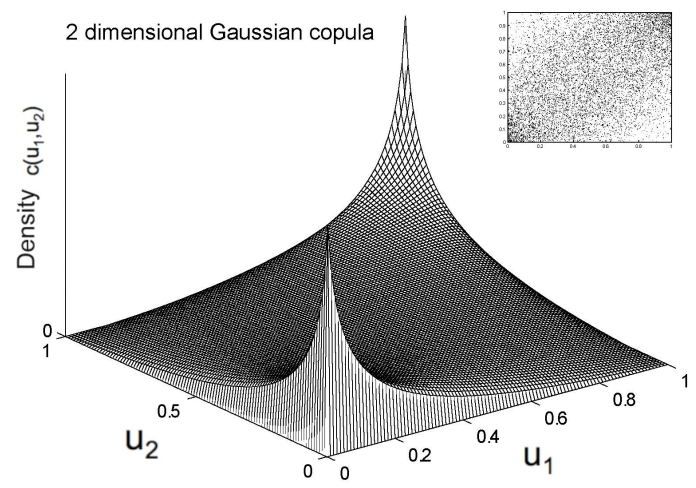

Fig. 3. 2-dimensional Gaussian copula density resulting from a sample of a bivariate standard Gaussian distribution with correlation coefficient $\rho=0.8$.

$$
\begin{aligned}
c\left(\Phi\left(x_{1}\right), \ldots, \Phi\left(x_{n}\right)\right) & =\frac{\frac{1}{(2 \pi)^{\frac{n}{2}}|R|^{\frac{1}{2}}} \exp \left(-\frac{1}{2} \mathbf{x}^{T} R^{-1} \mathbf{x}\right)}{\prod_{i=1}^{n} \frac{1}{\sqrt{(2 \pi)}} \exp \left(-\frac{1}{2} x_{i}^{2}\right)} \\
& =\frac{1}{|R|^{\frac{1}{2}}} \exp \left(-\frac{1}{2} \mathbf{x}^{T}\left(R^{-1}-I\right) \mathbf{x}\right),
\end{aligned}
$$

where $\mathbf{x}=\left(x_{1}, \ldots, x_{n}\right)$ and $\Phi(x)$ denotes the CDF of the standard Gaussian distribution. Equivalently, using $u_{i}=\Phi\left(x_{i}\right)$ we can write

$$
c\left(u_{1}, \ldots, u_{n}\right)=\frac{1}{|R|^{\frac{1}{2}}} \exp \left(-\frac{1}{2} \zeta^{T}\left(R^{-1}-I\right) \zeta\right),
$$

where $\zeta=\left(\Phi^{-1}\left(u_{1}\right), \ldots, \Phi^{-1}\left(u_{n}\right)\right)^{T}$.

For example, Figure 2 shows in the top right corner a random sample of a bivariate standard Gaussian distribution $f\left(x_{1}, x_{2}\right)$ with a Pearson correlation coefficient $\rho=0.8$. Using the transformation $u_{i}=F\left(x_{i}\right)$, we obtain the corresponding

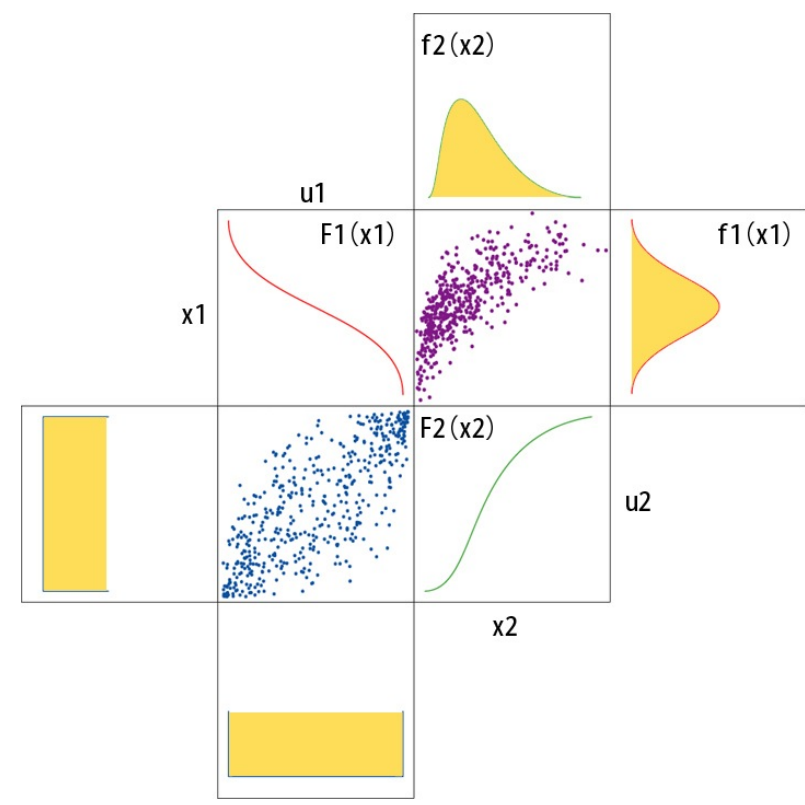

Fig. 4. Generation of a sample of a Gaussian copula from a sample of a bivariate distribution that has marginal distributions a Gamma and a Gaussian.

sample in the space $\left(u_{1}, u_{2}\right)$, which is shown in the bottom left corner. Figure 3 shows the surface of the Gaussian copula density $c\left(u_{1}, u_{2}\right)$.

Now consider two random variables $X_{1}$ and $X_{2}$ and suppose that $f_{1}\left(x_{1}\right)$ is a Gaussian distribution and $f_{2}\left(x_{2}\right)$ is a Gamma distribution, as shown in the top right corner of Figure 4. As before, using the transformation $u_{i}=F\left(x_{i}\right)$, we obtain the corresponding sample in the space $\left(u_{1}, u_{2}\right)$ as shown in the bottom left corner of Figure 4. Comparing Figures 2 and 4, it can be seen that the density $c\left(u_{1}, u_{2}\right)$ has the same parametric form as the Gaussian copula density, even though the marginal distribution $f_{1}\left(x_{1}\right)$ is a Gamma distribution. Moreover, the dependence between the uniform random variables $U_{1}$ and $U_{2}$ is linear, even though $X_{1}$ and $X_{2}$ appear to have a nonlinear dependence. In other words, the actual dependency between $X_{1}$ and $X_{2}$ is linear when the copulas-based scale-free model of dependence is considered.

The above example illustrates that Gaussian copulas can model the dependency between random variables that do not necessarily follow a Gaussian distribution. Next, we show how to simulate the Gaussian copula density.

\section{Simulation of Gaussian copulas}

Suppose that we have a representative sample $\mathbf{X}^{1}, \mathbf{X}^{2}, \ldots, \mathbf{X}^{m}$ of $\mathbf{X}$, with $\mathbf{X}^{j}=\left(X_{1}^{j}, X_{2}^{j}, \ldots, X_{n}^{j}\right)$, $j=1, \ldots, m$, which is mapped into the sample $\mathbf{U}^{1}, \mathbf{U}^{2}, \ldots, \mathbf{U}^{m}$ of $\mathbf{U}$, with $\mathbf{U}^{j}=\left(U_{1}^{j}, U_{2}^{j}, \ldots, U_{n}^{j}\right)$, $j=1, \ldots, m$, using the transformations $u_{i}=F_{i}\left(x_{i}\right)$. Suppose also that the resulting density $c\left(u_{1}, u_{2}, \ldots, u_{n}\right)$ corresponds to a Gaussian copula. Based on the sample $\mathbf{U}^{1}, \mathbf{U}^{2}, \ldots, \mathbf{U}^{m}$, our objective is to generate a sample of $\mathbf{U}$ of size $N, N \gg m$, which can be then easily mapped onto a corresponding sample of $\mathbf{X}$ using the inverse transformations $x_{i}=F_{i}^{-1}\left(u_{i}\right)$. 


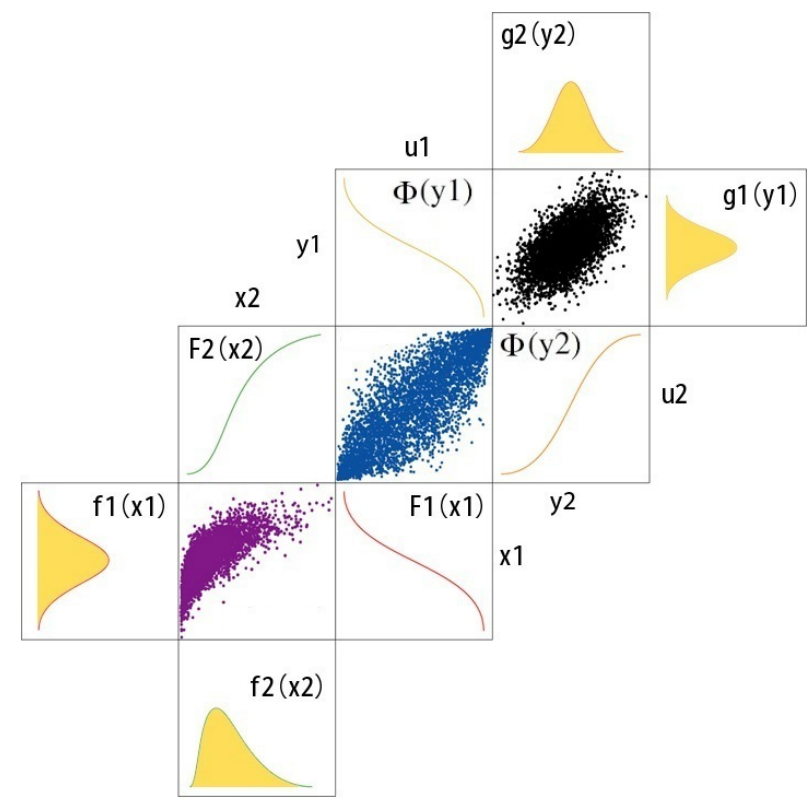

Fig. 5. Sample of size $10^{4}$ generated from a Gaussian copula.

For this purpose, we first apply the inverse transformations $y_{i}=\Phi^{-1}\left(u_{i}\right)$ on the sample $\mathbf{U}^{1}, \mathbf{U}^{2}, \ldots, \mathbf{U}^{m}$ to obtain a sample $\mathbf{Y}^{1}, \mathbf{Y}^{2}, \ldots, \mathbf{Y}^{m}$, with $\mathbf{Y}^{j}=\left(Y_{1}^{j}, Y_{2}^{j}, \ldots, Y_{n}^{j}\right)$, $j=1, \ldots, m$. By definition, each $Y_{i}$ follows a standard univariate Gaussian distribution, denoted by $g_{i}\left(y_{i}\right)$, and their joint PDF, denoted by $g\left(y_{1}, y_{2}, \ldots, y_{n}\right)$, is a standard multivariate Gaussian distribution with some correlation matrix $R$. Then, we fit $g\left(y_{1}, y_{2}, \ldots, y_{n}\right)$ by estimating its single parameter $R$ based on the available samples $\mathbf{Y}^{1}, \mathbf{Y}^{2}, \ldots, \mathbf{Y}^{m}$. The covariance of a pair $\left(Y_{i}, Y_{j}\right)$ is calculated using the sample Kendall's $\tau$ estimator given by Equation (1). Next, we simulate $g\left(y_{1}, y_{2}, \ldots, y_{n}\right)$ to generate $N$ samples of $\mathbf{Y}$ using a standard pseudo-random generator [11]. Finally, we use the transformations $u_{i}=\Phi\left(y_{i}\right)$ to map these samples to $N$ samples of $\mathbf{U}$.

The procedure is illustrated in Figure 5. Using the sample of size $m=10^{3}$ shown in the $\left(u_{1}, u_{2}\right)$ space of Figure 4 and the inverse transformations $y_{i}=\Phi^{-1}\left(u_{i}\right)$, we generated a corresponding sample of size $m=10^{3}$ in the $\left(y_{1}, y_{2}\right)$ space. This sample is used to fit a standard bivariate Gaussian distribution $g\left(y_{1}, y_{2}\right)$ which is thereafter simulated to obtain $N=10^{4}$ independent new samples in the space $\left(y_{1}, y_{2}\right)$ shown in the top right corner of Figure 5. Then, using the transformation $x_{i}=F_{i}^{-1}\left(\Phi\left(y_{i}\right)\right)$ we obtain the desired sample of size $N=10^{4}$ in the $\left(x_{1}, x_{2}\right)$ space shown in the bottom left corner of Figure 5. Notice that this sample follows the distribution of the original sample of size $m=10^{3}$ in the top right corner of Figure 4.

\section{E. Goodness-of-fit test for Gaussian copulas}

In this section, we discuss how to test the hypothesis that a sample $\mathbf{X}^{1}, \mathbf{X}^{2}, \ldots, \mathbf{X}^{m}$ of $\mathbf{X}$, with $\mathbf{X}^{j}=\left(X_{1}^{j}, X_{2}^{j}, \ldots, X_{n}^{j}\right)$, $j=1, \ldots, m$, which is drawn independently from a distribution $f\left(x_{1}, x_{2}, \ldots, x_{n}\right)$, results in a Gaussian copula. For this purpose, we will compare the empirical copula obtained based on the $m$ samples with the Gaussian copula. First, we show how to obtain the empirical copula.

To avoid introducing any assumptions on the marginal CDF $F_{i}\left(x_{i}\right)$, we will use the empirical CDF of $F_{i}\left(x_{i}\right)$, denoted by $\hat{F}_{i}\left(x_{i}\right)$, to transform the $m$ samples of $\mathbf{X}$ into $m$ samples of $\mathbf{U}$, denoted by $\hat{\mathbf{U}}^{j}=\left(\hat{U}_{1}^{j}, \hat{U}_{2}^{j}, \ldots, \hat{U}_{n}^{j}\right), j=1, \ldots, m$. The empirical CDF $\hat{F}_{i}\left(x_{i}\right)$ can be calculated as

$$
\hat{F}_{i}\left(x_{i}\right)=\frac{1}{m+1} \sum_{k=1}^{m} \mathbb{1}_{\left\{X_{i}^{k}<x_{i}\right\}} .
$$

The function $\mathbb{1}_{\{\text {arg }\}}$ is the indicator function, which equals 1 if $\arg$ is true and 0 otherwise. Here, $m+1$ is used to keep the empirical CDF lower than 1. Clearly, the empirical CDF converges towards the true one as $m \rightarrow \infty$. A sample of $\mathbf{U}$ can be obtained using $\hat{U}_{i}^{j}=\hat{F}_{i}\left(X_{i}^{j}\right)$, for $i=1, \ldots, n$ and $j=1, \ldots, m$. The empirical copula can then be described as

$$
\hat{C}\left(u_{1}, \ldots, u_{n}\right)=\frac{1}{m+1} \sum_{j=1}^{m} \mathbb{1}_{\hat{U}_{1}^{j} \leq u_{1}, \ldots, \hat{U}_{n}^{j} \leq u_{n}} .
$$

Several goodness-of-fit tests for copulas exist in the literature [16]. A specific test for the Gaussian copulas is presented in [17]. It relies on the remark that if the empirical copula in Equation (9) is Gaussian, then the samples $\hat{Y}_{i}^{j}=\Phi^{-1}\left(\hat{U}_{i}^{j}\right)$, $i=1, \ldots, n, j=1, \ldots, m$, of the random vector $\hat{\mathbf{Y}}=$ $\left(\hat{Y}_{1}, \hat{Y}_{2}, \ldots, \hat{Y}_{n}\right)$ must be necessarily drawn from a standard multivariate Gaussian distribution. To verify this necessary condition, we need to test the hypothesis that the random variable

$$
\hat{\mathbf{Z}}^{2}=\hat{\mathbf{Y}}^{T} \hat{\tau}^{-1} \hat{\mathbf{Y}}=\sum_{i=1}^{n} \sum_{j=1}^{n} \hat{Y}_{i} \hat{\tau}_{i j}^{-1} \hat{Y}_{j}
$$

where $\hat{\tau}_{i j}$ is obtained using Equation (1), follows a $\chi_{n}^{2}$ distribution with $n$ degrees of freedom [17]. This can be achieved using the univariate K-S test (see Section III-C).

As is indicated in [17], the above goodness-of-fit test for Gaussian copulas is very sensitive to the dimensionality $n$, with an increasing tendency to reject rather than to accept the null hypothesis as $n$ increases. Thus, the null hypothesis may be rejected just because of the high dimensionality of the data. Alternatively, it is possible to apply this test to each pair of random variables. This is justified as follows. Let us consider a triplet of random variables $\left(X_{1}, X_{2}, X_{3}\right)$ such that each pair of random variables, i.e. $\left(X_{1}, X_{2}\right),\left(X_{1}, X_{3}\right)$ and $\left(X_{2}, X_{3}\right)$, has a Gaussian copula. If the copula of the triplet $\left(X_{1}, X_{2}, X_{3}\right)$ is elliptical, then the triplet $\left(X_{1}, X_{2}, X_{3}\right)$ has also a Gaussian copula. This result generalizes to an arbitrary number of random variables. Since there is no test for ellipticity, the ellipticity property will be verified by simulation, as described in Section VI-C.

Finally, there are goodness-of-fit tests which can be applied for any type of copula [18].

\section{TEST METRICS ESTIMATION USING COPULAS}

The procedure for the estimation of parametric test metrics using a copulas-based statistical model is as follows. 


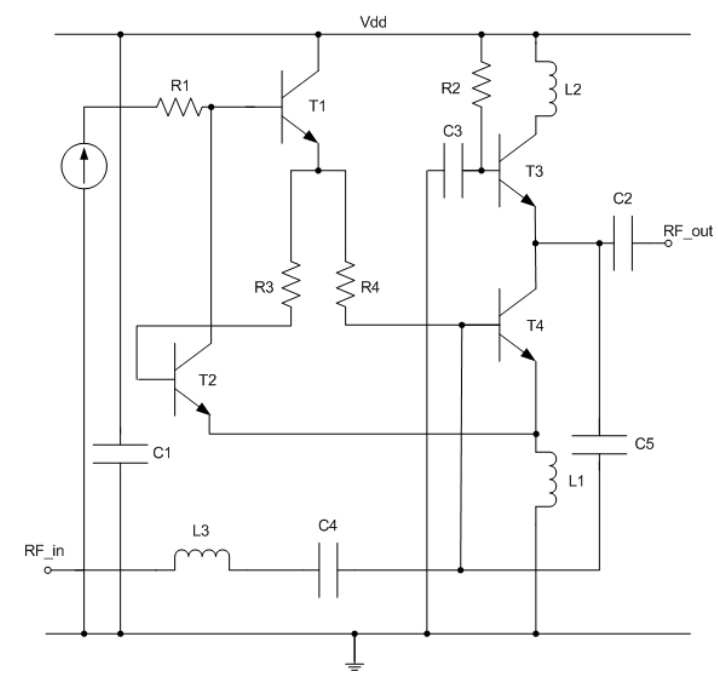

Fig. 6. LNA schematic.

1) Denote the vector of output parameters of the CUT as $\mathbf{X}=\left(X_{1}, X_{2}, \ldots, X_{n}\right)$ and run $m$ circuit-level Monte Carlo simulations to obtain $m$ samples of $\mathbf{X}$.

2) Use a goodness-of-fit test to verify that the $m$ samples of $\mathbf{X}$ result in a Gaussian copula, as explained in Section IV-E.

3) Simulate the Gaussian copula and generate $N, N \gg m$, new observations of $\mathbf{X}$, as explained in Section IV-D.

4) Given the test limits and the performance specifications, calculate the test metrics based on relative frequencies using the generated large sample of $\mathbf{X}$, as explained in the introduction.

\section{EXAMPLE}

In this Section, we use the proposed method to evaluate a BIT measurement for an RF LNA. In particular, we show how to generate a large sample of LNA instances using the copulasbased statistical model. The generated sample is used to fix the test limits on the BIT measurement, in order to achieve desired trade-offs between the defect level and the yield loss.

\section{A. Test vehicle}

As a case study, we consider the evaluation of a BIT technique for a $1.9 \mathrm{GHz}$ cascode LNA which is designed in a 0.25 $\mu m$ BiCMOS STMicroelectronics technology. The schematic of the LNA is shown in Figure 6. Table I summarizes the performances of the LNA and their specifications. The BIT measurement is a DC signature obtained by computing the RMS value of the cross-correlation between the output voltage and the power supply current of the LNA when a $1.9 \mathrm{GHz}$ sinusoidal stimulus with magnitude $-30 \mathrm{dBm}$ is applied at its input [19]. The output parameter vector $\mathbf{X}$ includes the five circuit performances (e.g. NF, $S_{11}$, Gain, 1-dB CP, and IIP3) and the BIT measurement.

\section{B. Validation of the Gaussian copula hypothesis}

As a first step, we have performed a Monte Carlo circuitlevel simulation of 1000 instances. The matrix plot of Figure 7
TABLE I

LIST OF SPECIFICATIONS FOR THE LNA.

\begin{tabular}{|l|l|l|l|}
\hline $\mathrm{N}^{\circ}$ & Performance & \multicolumn{2}{|c|}{ Specifications } \\
\cline { 3 - 4 } & & 1ower limit & upper limit \\
\hline \hline 1 & NF & $-\infty$ & $1.3 \mathrm{~dB}$ \\
2 & $\mathrm{~S}_{11}$ & $-\infty$ & $-9 \mathrm{~dB}$ \\
3 & Gain & $17 \mathrm{~dB}$ & $+\infty$ \\
4 & $1-\mathrm{dB}$ CP & $-11.3 \mathrm{dBm}$ & $+\infty$ \\
5 & IIP3 & $-5.1 \mathrm{dBm}$ & $+\infty$ \\
\hline
\end{tabular}

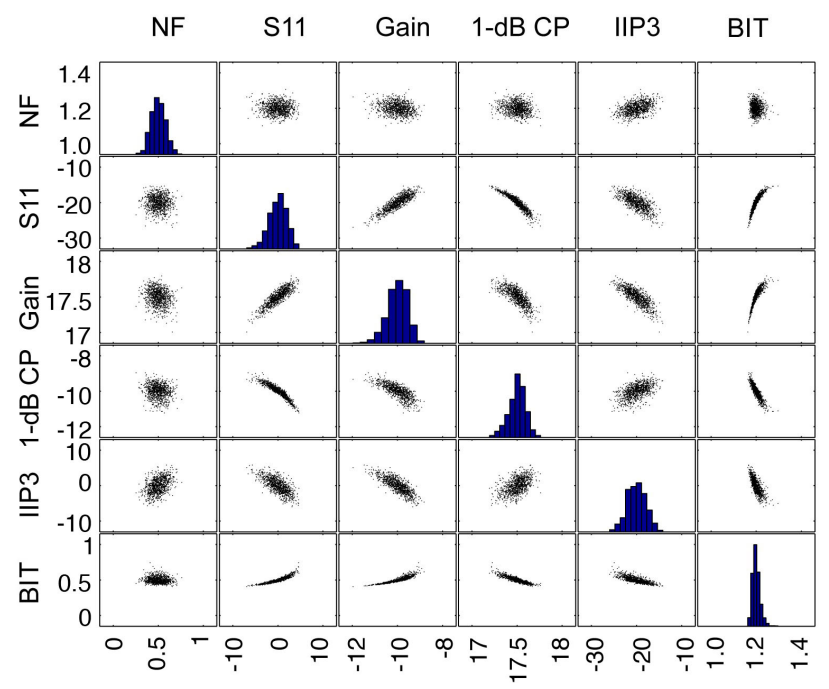

Fig. 7. Initial sample of the LNA circuit obtained using Monte Carlo circuit level simulation.

shows in the diagonal the histograms of each output parameter. The rest of the matrix shows the bivariate distributions of each pair of output parameters.

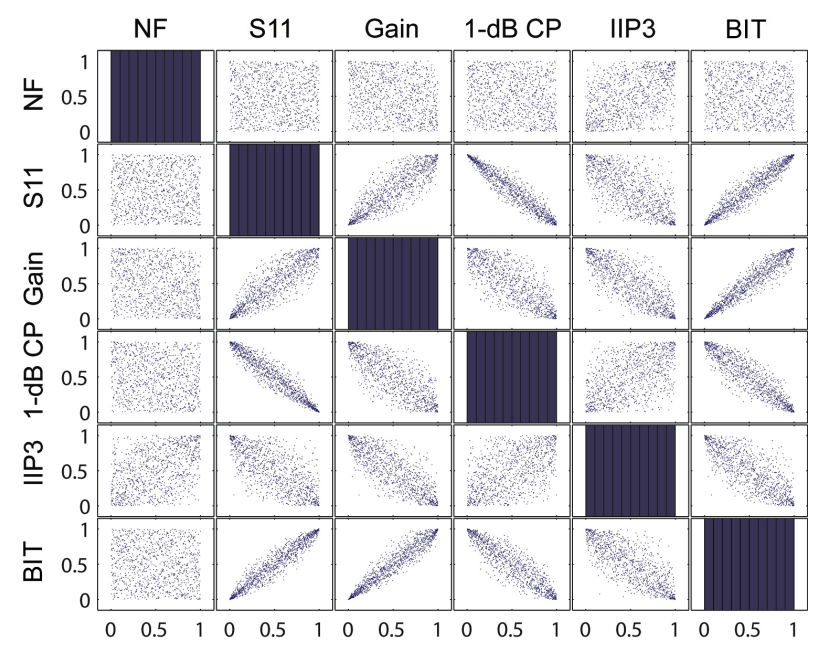

Fig. 8. Empirical copula of the initial data of the LNA.

Next, for each pair of output parameters in Figure 7, we generate the corresponding copula samples using the empirical univariate $\operatorname{CDFs} \hat{F}_{i}\left(x_{i}\right)$, similarly to Figures 2 and 4 in Section IV-C. The copula samples are shown in Figure 8. Comparing each bivariate copula distribution in Figure 8 to the bivariate Gaussian copula distribution in Figure 3, we can observe that 


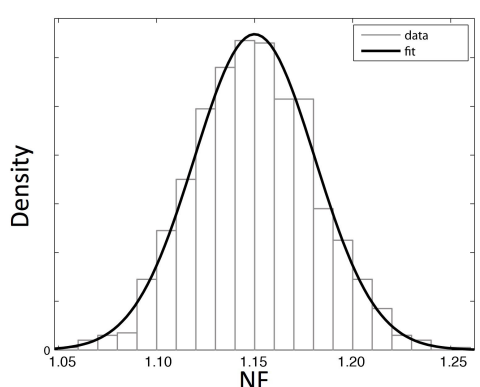

(a)

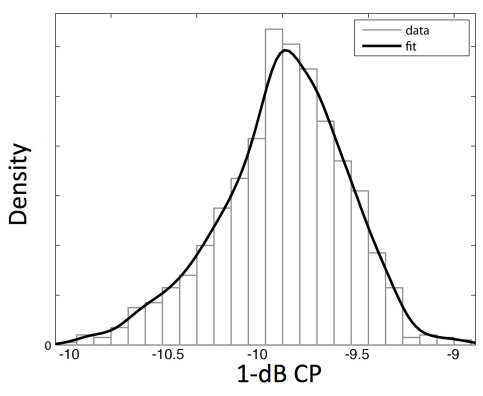

(d)

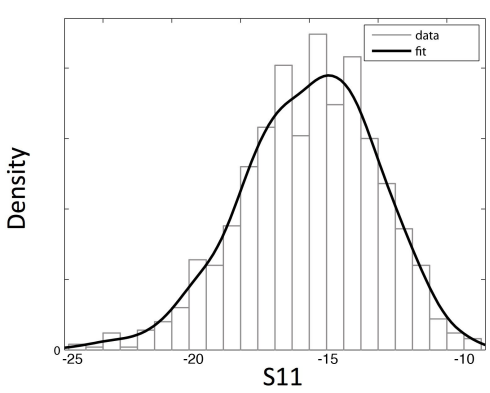

(b)

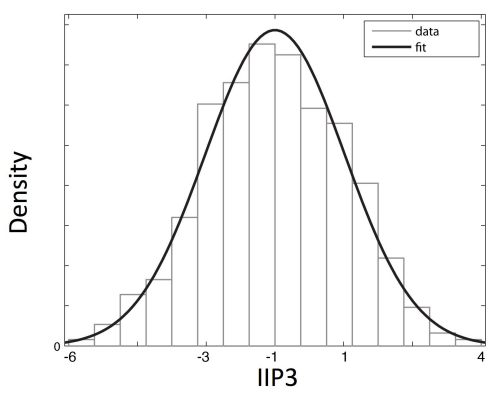

(e)

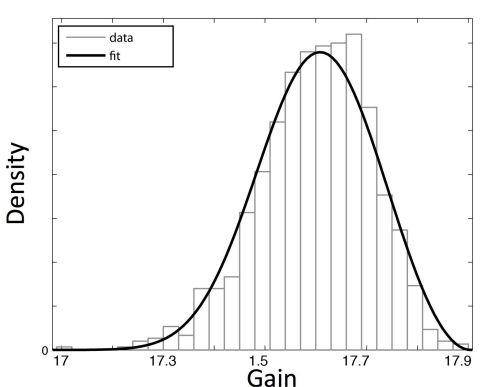

(c)

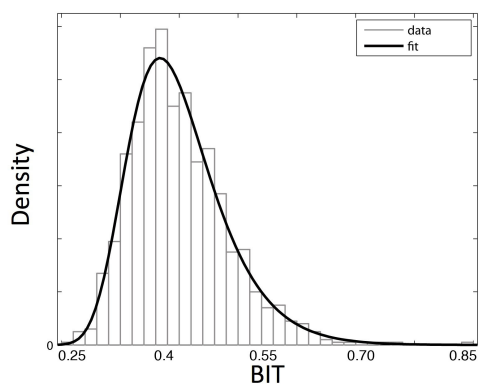

(f)

Fig. 9. Fitted distributions of the output parameters.

they have the same parametric form, with the difference being in the spread of the samples across one diagonal. Different spreads are explained by the different level of dependency existing in each pair.

In order to formally verify that each bivariate copula is indeed a Gaussian copula, we use the goodness-of-fit test presented in Section IV-E. This test supports the hypothesis that, for each pair of output parameters, the corresponding variable $\hat{\mathbf{Z}}^{2}$ follows a $\chi_{2}^{2}$-distribution. Thus, the copula of each pair of output parameters can be assumed Gaussian. Now, to verify that the global copula is Gaussian, we need to prove that it is also elliptical. To this end, we generate a sample from a standard multivariate Gaussian distribution that has the same correlation matrix as the sample of the empirical copula. The generated sample is transformed to a sample in the copula space using the standard univariate CDFs. Then, we test if this sample and the sample of the empirical copula originate from the same distribution by using the K-S test. This test supports the hypothesis that the global copula is elliptical and, thereby, we conclude that the global copula is indeed Gaussian. We notice that we have used the goodness-of-fit test in [17] because of its practical implementation and ease of understanding. In addition, we have also used the test in [18] which can be applied to any type of copula. This test does not reject either the null hypothesis that the empirical copula is Gaussian with a confidence level of $95 \%$.

\section{Generation of statistical data}

In this step, we simulate the Gaussian copula to obtain $N=10^{6}$ samples of the output parameters, as explained in Section IV-D with the help of Figure 5. For this purpose, we
TABLE II

PARAMETERS OF THE FITTED MARGINAL DISTRIBUTIONS.

\begin{tabular}{|l||l|l|l|l|l|}
\hline \multirow{2}{*}{} & Fitted & \multicolumn{4}{|c|}{ Parameters } \\
\cline { 3 - 6 } & Distribution & $\mu$ & $\sigma$ & Bandwidth & $\xi$ \\
\hline \hline NF & Gaussian & 0 & 0.03 & - & - \\
S $_{11}$ & NP & - & - & 0.4 & - \\
Gain & GEV & -0.036 & 0.116 & - & -0.34 \\
1-dB CP & NP & - & - & 0.089 & - \\
IIP3 & Gaussian & 0 & 1.014 & - & - \\
\hline BIT & GEV & -0.018 & 0.034 & - & -0.049 \\
\hline
\end{tabular}

need to first estimate the marginal distributions of the output parameters. Figure 9 shows the fitted marginal distributions together with the corresponding histograms. In Table II, we list the type of the fitted marginal distributions, as well as their parameters. GEV refers to the Generalized Extreme Value distribution which is defined based on three parameters, namely the location parameter $\mu$, the scale parameter $\sigma$, and the shape parameter $\xi$. NP refers to a nonparametric distribution obtained using KDE which is fully described by the type of the kernel used and the bandwidth. Here, we have used the Gaussian kernel. Each fitted marginal distribution has been validated using the K-S test (see Section III-C).

As an illustrative example, Figures 10 and 11 show respectively a simulated sample of size $10^{4}$ of the Gaussian copula and the resulting corresponding sample of the output parameters. The benefit of the method can be deduced by comparing the original simulation data in Figure 7 with the generated synthetic data in Figure 11. Starting from observed data corresponding to 1000 circuit simulations, we have succeeded to generate in a few seconds more data that are identically distributed. Using the copulas-based statistical model, it takes 


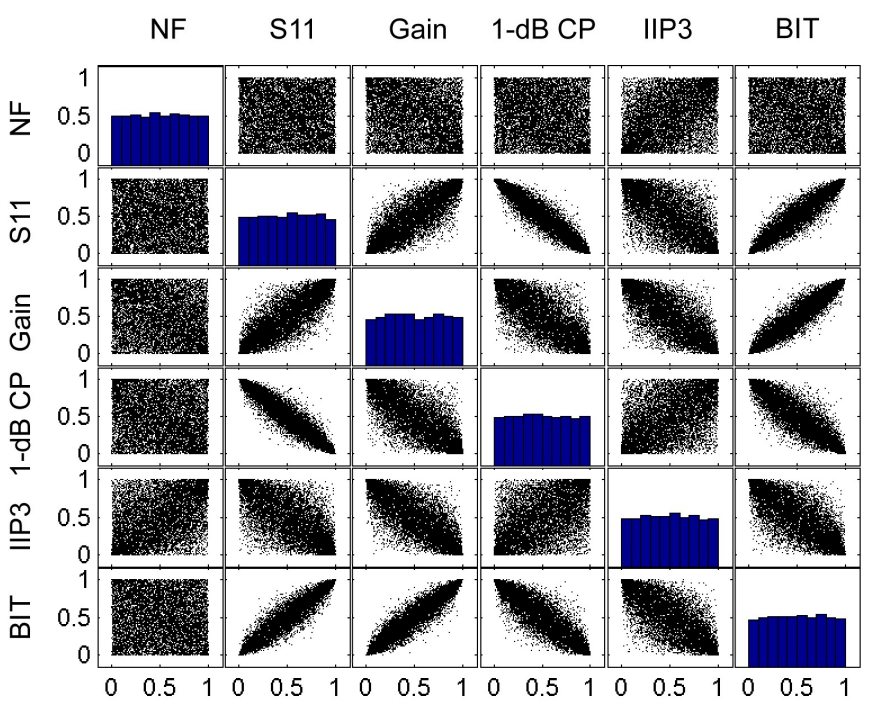

Fig. 10. Sample of the fitted Gaussian copula of size $10^{4}$

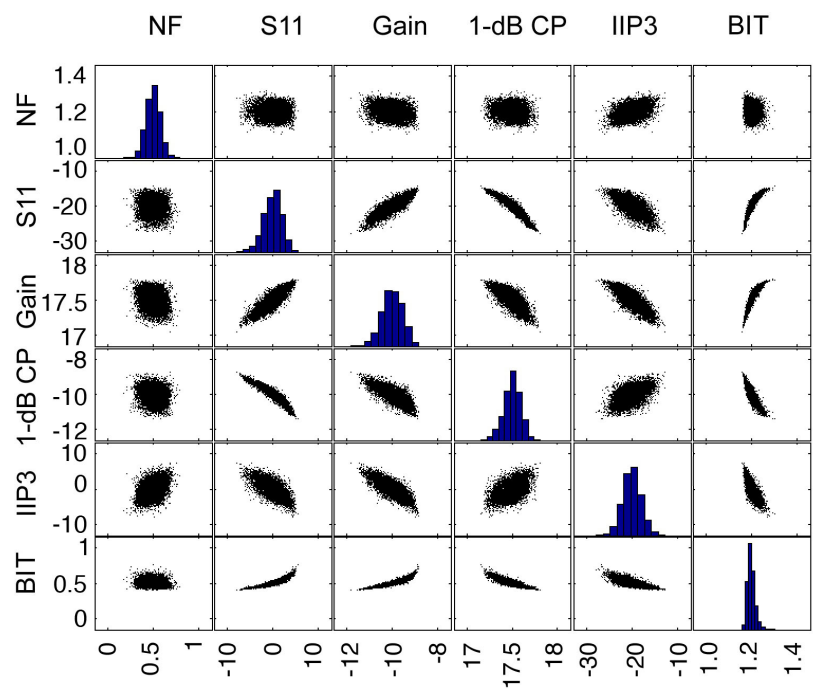

Fig. 11. Sample of LNA output parameters of size $10^{4}$.

only a few seconds to generate new data corresponding to $N=10^{6}$ circuit instances.

\section{Setting test limits}

Given a lower and upper test limit for the BIT measurement, we can compute the resulting defect level $(D L)$ and yield loss $\left(Y_{L}\right)$ based on the large sample generated with the copulasbased statistical model. The defect level and yield loss move in opposite directions as we change the test limits. Strict test limits result in low defect level and large yield loss. Lenient test limits result in large defect level and low yield loss. We observed that the optimal test limits should lie somewhere within the interval $[0.05,0.75]$. We set out to find the best tradeoff by calculating the defect level and yield loss for different test limits [0.35-0.01k,0.45+0.01k], where $k$ runs from 0 to 30 with a step of 1 . The trade-off is shown in Figure 12. Notice that the generated large sample has $49 \mathrm{dppm}$. Different criteria

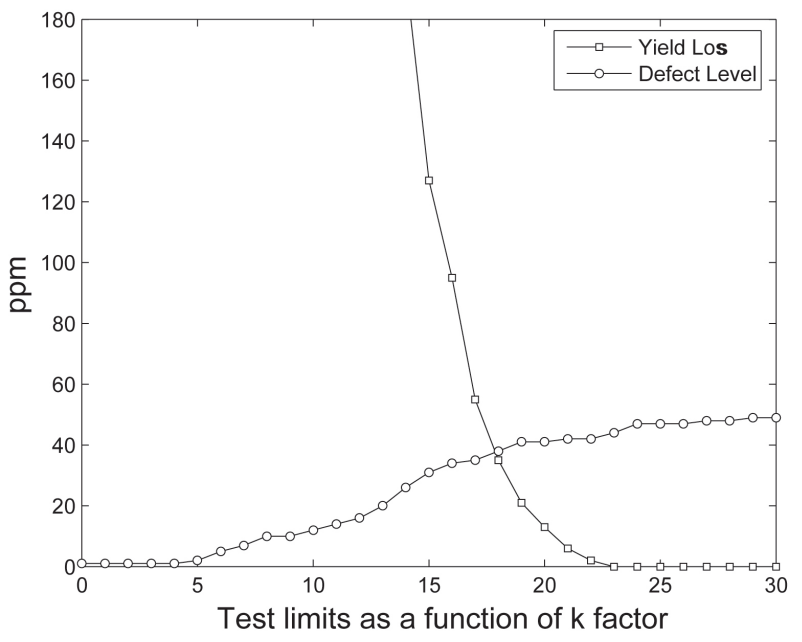

Fig. 12. Test limits as a function of k vs. test metrics $Y_{L}$ and $D L$.

TABLE III

BIT LIMITS CALCULATED USING THE COPULAS-BASED DENSITY ESTIMATION FOR DIFFERENT TRADE-OFFS BETWEEN DEFECT LEVEL AND YIELD LOSS.

\begin{tabular}{|l|c|c|}
\hline Case & BIT limits & $D L$ \\
\hline$Y_{L}=D L$ & {$[0.161,0.638]$} & 37 \\
$Y_{L}=10 \cdot D L$ & {$[0.204,0.596]$} & 23 \\
\hline
\end{tabular}

can be used for setting the test limits. We can set the yield loss equal to the defect level or we can apply the rule-of-ten which suggests that it is ten times more expensive to ship to the customer a faulty device than to reject a functional one. The resulting test limits and test metrics for the above two criteria are shown in Table III.

\section{ViI. Choosing the Most Appropriate Method}

\section{A. Comparison with previously proposed methods}

In this section, we apply two previously proposed techniques for generating a sample of synthetic LNA instances [2], [3]. These techniques rely on simulating the density $f\left(x_{1}, x_{2}, \ldots, x_{n}\right)$ of the output parameters, as explained in the introduction and in Section II. The density is fitted using the initial data from circuit-level Monte Carlo simulation. In [2], the density is assumed to be Gaussian, whereas, in [3], the density is considered to have a nonparametric form. The resulting sample distributions are compared to the one obtained by applying the copulas theory.

Let us consider without loss of generality the pair $S_{11}$-BIT. Figure 13(a) shows the sample distribution obtained by the copulas-based model. This Figure is a zoom out of the box $S_{11}$-BIT of Figure 11. In addition, the initial Monte Carlo data shown in the corresponding box of Figure 7 are superimposed. It can be seen that the two sample distributions match, which clearly demonstrates the accuracy of the copulas-based model. Figure 13(b) shows the sample distribution obtained by fitting a Gaussian density. As expected, this distribution has an elliptical shape and, thus, it is a crude approximation to the original banana shape. Therefore, a Gaussian density is deemed insufficient to model the relationship between $S_{11}$ 


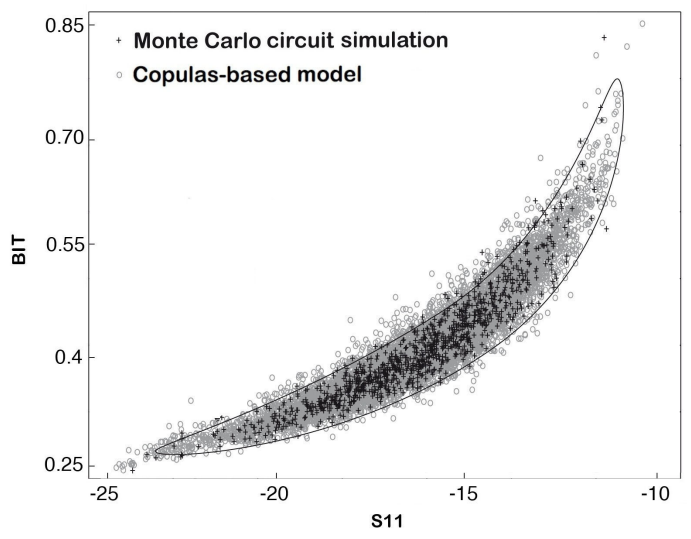

(a)

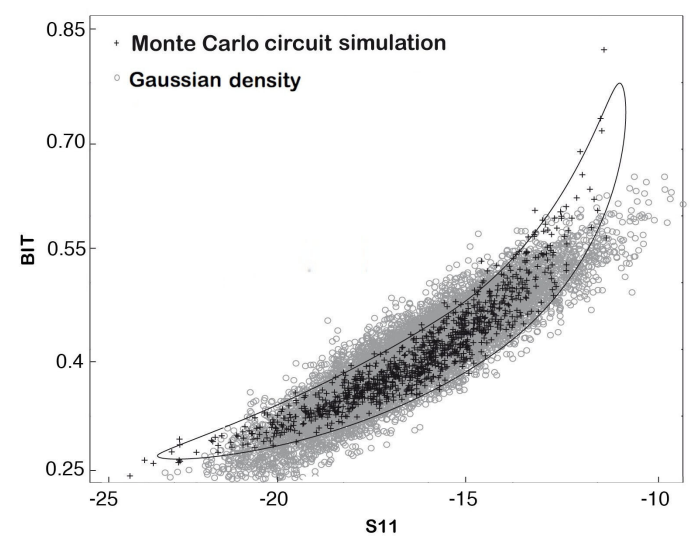

(b)

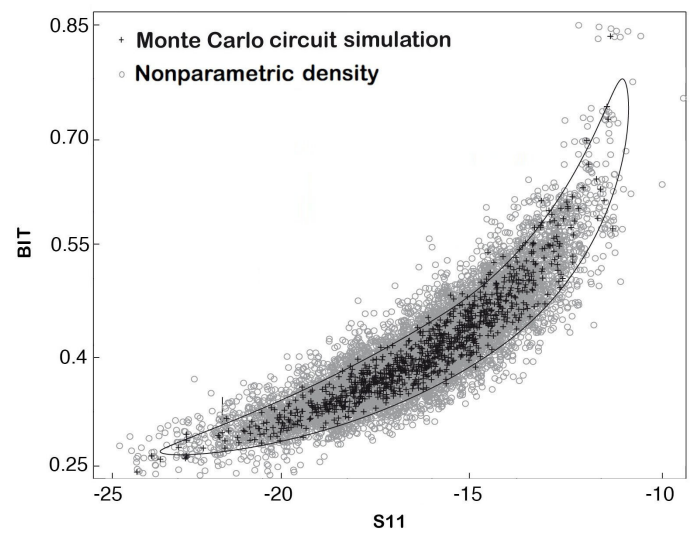

(c)

Fig. 13. Comparison of a sample distribution of size $10^{4}$ of the output parameters $S_{11}$ and BIT obtained by using: (a) the copulas-based model, (b) a multivariate Gaussian density [2], and (c) a nonparametric density [3]. Each statistical model is built using the same data generated by 1000 circuit-level Monte Carlo simulations.

and BIT. Figure 13(c) shows the result when a nonparametric density is used. Although the shape of the joint distribution is well approximated, we observe a larger dispersion of the generated data with respect to the copulas-based model.

The dppm values for the three methods, calculated based on $N=10^{6}$ synthetic circuit instances, are listed in Table IV. As discussed above with the help of Figure 13(b), it
TABLE IV

DPPM OF THE CUT CALCULATED BY DIFFERENT DENSITY ESTIMATION METHODS.

\begin{tabular}{|c|c|c|c|}
\cline { 2 - 4 } \multicolumn{1}{c|}{} & copulas & Gaussian density & nonparametric density \\
\hline $\mathrm{dppm}$ & 49 & 100 & 1020 \\
\hline
\end{tabular}

is inaccurate to fit a Gaussian density, thus we should not trust the corresponding estimated dppm value of 100. From Table IV, it can be observed that the nonparametric density method results in $1020 \mathrm{dppm}$, a value that is significantly larger compared to the $49 \mathrm{dppm}$ that result from the copulasbased model. The value of $49 \mathrm{dppm}$ is probably closer to reality since this particular LNA is an industrial design. With this in mind, it appears that the nonparametric density method has overestimated the probability at the tails of the distribution.

\section{B. Recommendations}

We can give the following recommendations as to which method to use in different scenarios. If the joint PDF $f\left(x_{1}, x_{2}, \ldots, x_{n}\right)$ of the output parameters belongs to a known family of distributions, then it is best to fit $f\left(x_{1}, x_{2}, \ldots, x_{n}\right)$ to this distribution and, subsequently perform probability sampling to obtain the synthetic data. If $f\left(x_{1}, x_{2}, \ldots, x_{n}\right)$ appears to have a nonparametric form, then we should examine first whether the resulting copula $C\left(u_{1}, u_{2}, \ldots, u_{n}\right)$ has a known parametric form. In this paper, we studied Gaussian copulas, but it should be stressed that there are other copula functions as well, such as the Student copula which results from a Student's $\mathrm{t}$-distribution. If a known copula is recognized, then it is best to use the method outlined in this paper. Finally, if the copula itself has an unknown form, then it is best to use the more general method based on nonparametric density estimation.

\section{CONCLUSIONS}

The estimation of test metrics such as parametric defect level and yield loss is a crucial task for adopting a builtin test technique for mixed-signal/RF circuits early at the design stage. Probability density estimation of the output parameters (e.g. performances and test measurements) offers a practical approach for estimating these metrics. This work has presented a new approach for parametric test metrics estimation using the copulas theory. The copulas-based model separates the dependencies between the output parameters from their marginal distributions, providing a complete and scale-free description of dependence that is more suitable to be modeled using well-known multivariate parametric laws. Using a built-in test technique for an RF LNA as a casestudy, we have shown that the new approach is more accurate than previous approaches, circumventing limitations that arise due to the arbitrary form of the output parameter distributions and the dimensionality of the output space. In addition, this method is very fast and simple to implement and it has been included in an existing mixed-signal/RF Computer-Aided-Test (CAT) platform [20]. 


\section{REFERENCES}

[1] A. Bounceur, and S. Mir, Estimation of test metrics for AMS/RF BIST using copulas, In IEEE 14th International Mixed-Signals, Sensors, and Systems Test Workshop, Vancouver, Canada, 2008.

[2] A. Bounceur, S. Mir, E. Simeu, and L. Rolíndez. Estimation of test metrics for the optimisation of analogue circuit testing. Journal of Electronic Testing: Theory and Applications, Springer Science+Business Media, 23(6), 2007, pp. 471-484.

[3] H. Stratigopoulos, S. Mir, and A. Bounceur. Evaluation of analog/RF test measurements at the design stage. IEEE Transactions on ComputerAided Design of Integrated Circuits and Systems, 28(4), April 2009, pp. 582-590.

[4] L. Milor, and A. Sangiovanni-Vincentelli, Computing parametric yield accurately and efficiently. IEEE International Conference on ComputerAided Design, Santa Clara, USA, 1990, pp. 116-119.

[5] C.M. Kurker, J.J. Paulos, R.S. Gyurcsik, and J.-C. Lu. Hierarchical yield estimation of large analog integrated circuits. IEEE journal of solid-state circuits, 28(3), March 1993, pp. 203-209.

[6] R.-B. Nelsen. An Introduction to Copulas. Lecture Notes in Statistics. Springer, New York, 1999.

[7] H. Abdi. Kendall rank correlation. In Encyclopedia of Measurement and Statistics. N. Salkind (Ed.). Sage Publications, 2007.

[8] C. Spearman. The proof and measurement of association between two things. Amer. J. Psychol., 15 (1904), pp. 72-101.

[9] C. Croux, and C. Dehon. The influence function of the Kendall rank correlation. Research report. Catholic University of Leuven.

[10] Matlab Software. The Language of Technical Computing.

[11] S. Kotz, N. Balakrishnan, and N.-L. Johnson. Continuous Multivariate Distributions: Models and Applications. John Wiley \& Sons Inc, (2000).

[12] A. Papoulis, Probability, Random Variables, and Stochastic Processes. Third edition. McGraw-Hill, 2001.

[13] B. W. Silverman, Density Estimation for Statistics and Data Analysis. London, U.K.: Chapman \& Hall, 1986.

[14] A. Sklar. Fonctions de répartition à $n$ dimensions et leurs marges. Publ. Inst. Statist. Université de Paris 8, 1959, pp. 229-231.

[15] J. Yan. Enjoy the Joy of Copulas: With a Package copula. In Journal of Statistical Software, 21(4), October 2007. http://www.jstatsoft.org/.

[16] C. Genest, B. Rémillad, and D. Beaudoin. Goodness-of-fit tests for copulas: A review and a power study. In Insurance: Mathematics and Economics, Elsevier, Volume 44, pp. 199-213, 2009.

[17] Y. Malevergne. Testing the Gaussian Copula Hypothesis for Financial Assets Dependences. In Quantitative Finance, Volume 3, pp. 231-250 (2003).

[18] V. Panchenko. Goodness-of-fit test for copulas. In Physica A 355, 176182.

[19] J. Machado da Silva, Low-power in-circuit testing of a LNA, In IEEE International Mixed-Signals Testing Workshop, 2005, pp. 206-210.

[20] A. Bounceur, S. Mir, L. Rolíndez, and E. Simeu. CAT platform for analogue and mixed-signal test evaluation and optimization, 2007, in VLSI-SoC : Research trends in VLSI and Systems on Chip, eds. De Micheli, G., Mir, S., Reis, R., Eds., Springer, Boston, pp. 281-300.

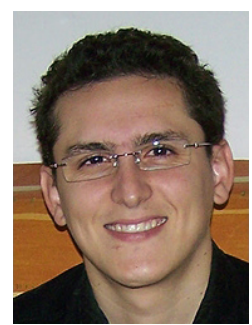

Ahcène Bounceur ( $\left.S^{\prime} 07\right)$ received the Engineer degree in Operations Research from the Bejaia University, Algeria, in 2002, the Ms.C. degree in Operations Research, Combinatorial and Optimization from the École Nationale Supérieure d'Informatique et de Mathématiques Appliquées de Grenoble (ENSIMAG), France, in 2003, and the Ph.D. degree in Micro and Nano electronics from Institut National Polytechnique de Grenoble, France, in 2007. Currently, he is an associate professor at the Université de Bretagne Occidentale, Brest, France. His current research interests are in the area of CAT tools development for analogue and mixed-signal testing.

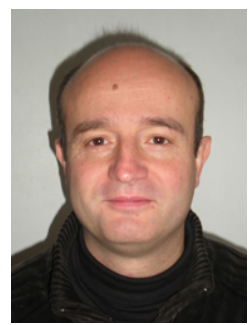

Salvador Mir (M'99) has an Industrial Engineering (Electrical, 1987) degree from the Polytechnic University of Catalonia, Barcelona, Spain, and M.Sc. (1989) and Ph.D. (1993) degrees in Computer Science from the University of Manchester, UK. He is a Research Director of CNRS (Centre National de la Recherche Scientifique) at TIMA Laboratory in Grenoble, France. He is currently Vice-director of TIMA and he is leading the RMS (Reliable Mixedsignal Systems) Group. He has published many papers in the field of mixed-signal/RF/MEMS test and he is editor of two books on silicon Microsystems. He has been General Chair of the IEEE Mixed-Signals Testing Workshop (IMSTW) in 2005 and the IFIP/IEEE International Conference on Very Large Scale Integration (VLSISoC) in 2006.

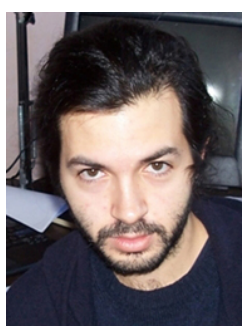

Haralampos-G. Stratigopoulos (S'02-M'07) received the Diploma in electrical and computer engineering from the National Technical University of Athens, Athens, Greece, in 2001 and the Ph.D. in electrical engineering from Yale University, New Haven, CT, in 2006. He is currently a Researcher with the French National Research Council (CNRS), TIMA Laboratory, Grenoble, France. His research interests are in the area of mixed-signal/RF design and test, machine learning, and neuromorphic VLSI circuits. 